\title{
SOME EXACT SOLUTIONS OF BURGERS-TYPE EQUATIONS*
}

\author{
By P. L. SACHDEV (Indian Institute of Science, Bangalore)
}

\begin{abstract}
A class of Burgers-type equations, reducible through a generalized Hopf-Cole transformation to a linear diffusion equation, are treated by similarity methods. New exact solutions of these equations are obtained and related to the wellknown solutions of the standard Burgers equation. Physical applications of these solutions are indicated.
\end{abstract}

1. Introduction. The well-known Burgers equation has been treated by many authors with reference to varied physical phenomena which it represents. An exhaustive compilation of its solutions and applications has been done recently by Benton and Platzman [1] (B \& P henceforth). However, very often, the equations that arise subject to various approximations do not conform to the standard Burgers equation. The additional terms arise from geometrical expansion [2], inhomogeneity of the medium [3], [4] or stronger nonlinearity [5]. These equations generally do not admit exact solutions. The purpose of this paper is to give a large class of self-similar solutions of inhomogeneous Burgers-like equations. Since the corresponding linear diffusion equation (to which these equations reduce) does not admit a constant solution, solutions similar to the "allied groups" of B \& P are unfortunately not possible. However, we do indicate how more general exact solutions may be constructed from the similarity solutions. These serve to give qualitative behavior of the solutions subject to general initial boundary conditions and may provide a check on the numerical [2] or perturbation [6] solutions of the more complicated equations of the same type. Rodin [7] has found solutions of an inhomogeneous Burgers equation through a very special similarity transformation. His solutions are included in our larger class of solutions.

2. Reduction of the generalized Burgers equations to a linear form. Chu [8] has considered the system of $n$ equations

$$
\frac{\partial u_{i}}{\partial t}+F_{i} \frac{\partial u_{i}}{\partial x_{i}}=G_{i} \frac{\partial u_{i}}{\partial x_{i}} \frac{\partial u_{i}}{\partial x_{i}}+\frac{\delta \partial^{2} u_{i}}{\partial x_{i} \partial x_{i}}+H_{i} R_{i}
$$

where the summation convention is adopted but the index $i$ is not summed, $F_{i}, G_{i}$ and $H_{i}$ are at least twice continuously differentiable functions of $u_{i}, \delta$ is a constant and $R_{i}$ are continuously differentiable functions of $t, x_{1}, x_{2}, \cdots, x_{n}$. Under the HopfCole-like transformations

$$
F_{i}\left(u_{i}\right)=\frac{2 \delta}{\theta} \frac{\partial \theta}{\partial x_{i}},
$$

* Received December 31, 1974. 
the system (2.1) reduces, after an integration, to the linear heat equation,

$$
\frac{\partial \theta}{\partial t}=\delta \frac{\partial^{2} \theta}{\partial x_{i}} \frac{\partial x_{i}}{}+\left[C(t)+\frac{P}{2 \delta}\right] \theta,
$$

if the functions $F_{i}, G_{i}$ and $H_{i}$ are derivable from a generating function $f_{i}\left(u_{i}\right)$ through

$$
\begin{aligned}
F_{i} & =\int^{u_{i}} f_{i}(u) d u, \\
G_{i} & =\delta \frac{d \ln f_{i}}{d u_{i}}, \\
H_{i} & =f_{i}^{-1},
\end{aligned}
$$

(where $i$ is not summed) and the function $P(x, t)$ is given by

$$
R_{i}=-\partial P / \partial x_{i}
$$

In the above, $C(t)$ is an arbitrary function of $t$ arising out of an integration with respect to $x$ of the transformed equation.

We shall be concerned only with a single equation in this paper rather than a system. Thus, once the generating function $f$ is chosen, the original nonlinear equation can be constructed. For example, for $f=0,1, e^{u}, n u^{n-1}, \ln u,-\sin u$, and $\cos u$ respectively, we get the following variety of equations:

$$
\begin{gathered}
\frac{\partial u}{\partial t}=\delta \frac{\partial^{2} u}{\partial x^{2}} \\
\frac{\partial u}{\partial t}+u \frac{\partial u}{\partial x}=\delta \frac{\partial^{2} u}{\partial x^{2}}+R(t, x) \\
\frac{\partial u}{\partial t}+e^{u} \frac{\partial u}{\partial x}=\delta\left(\frac{\partial u}{\partial x}\right)^{2}+\delta \frac{\partial^{2} u}{\partial x^{2}}+e^{-u} R(t, x), \\
\frac{\partial u}{\partial t}+u^{n} \frac{\partial u}{\partial x}=\delta \frac{n(n-1)}{u}\left(\frac{\partial u}{\partial x}\right)^{2}-\delta \frac{\partial^{2} u}{\partial x^{2}}+\frac{R(t, x)}{n u^{n-1}}, \\
\frac{\partial u}{\partial t}+u(\ln u-1) \frac{\partial u}{\partial x}=\frac{\delta}{u \ln u}\left(\frac{\partial u}{\partial x}\right)^{2}+\delta \frac{\partial^{2} u}{\partial x^{2}}+\frac{R(t, x)}{\ln u}, \\
\left.\begin{array}{c}
\cos u \\
\sin u
\end{array}\right) \frac{\partial u}{\partial x}=-\delta\left(\begin{array}{c}
\cot u \\
\tan u
\end{array}\right)\left(\frac{\partial u}{\partial x}\right)^{2}+\delta \frac{\partial^{2} u}{\partial x^{2}}+\left(\begin{array}{c}
-\operatorname{csu} u \\
\sec u
\end{array}\right) R(t, x) .
\end{gathered}
$$

Here $R(t, x)$ is any function integrable with respect to $x$. It is interesting to note that even for $R=0$, the homogeneous heat equation provides solutions for a large class of equations (2.6), different from Burgers. However, we shall be mainly interested in the case, $R \neq 0$, and hence in Eq. (2.3).

3. Some exact solutions. One observes from Tables 1, 2 and 3 of B \& P (to which we refer all along) that the solutions of the heat equation enumerated are either product solutions or similarity solutions and their linear superpositions. In what follows we shall derive such solutions of Eq. (2.3). We note that these solutions are independent 
of the form of the generating function $f$ and, therefore, cover the whole class that is generated by any admissible $f$.

Absorbing $C(t)$ in $P(x, t)$ and changing the variables according to

$$
\frac{x}{L} \rightarrow \bar{x}, \quad \frac{\delta t}{L^{2}} \rightarrow \bar{t}, \quad \frac{u L}{\delta} \rightarrow \bar{u}, \quad P(x, t) \rightarrow \frac{2 \delta^{2}}{L^{2}} \bar{P}(\bar{x}, \bar{t})
$$

where $L$ is characteristic length of the wave phenomenon being considered, we can write Eq. (2.3) as

$$
\frac{\partial \theta}{\partial t}=\frac{\partial^{2} \theta}{\partial x^{2}}+P(x, t) \theta
$$

wherein the bars have been dropped.

a) Similarity solutions. Assuming $\theta=t^{n} \psi(z)$ where $z=x^{2} / 4 t$ and $n$ is any real number, Eq. (3.2) becomes an ordinary differential equation in $z$ if the function $P$ is of the form

$$
P(x, t)=\frac{1}{t} Q(z)
$$

$\psi$ satisfies the equation

$$
z \psi^{\prime \prime}+\left(z+\frac{1}{2}\right) \psi^{\prime}+(Q(z)-n) \psi=0 .
$$

This equation can be easily treated. We consider some special cases.

(i) If $Q(z)=z / 4$, Eq. (3.4) admits the solution

$$
\psi=e^{-z / 2}\left(C_{1}+C_{2} z^{1 / 2}\right)
$$

where $C_{1}$ and $C_{2}$ are arbitrary constants.

Choosing $C_{1}=1$ and $C_{2}=0$, we have $\theta=t^{-1 / 4} e^{-z / 2}$. The corresponding function $u=x / 2 t$ solves the equation

$$
u_{t}+u u_{x}=u_{x x}-\left(x / 4 t^{2}\right)
$$

and is the saw-tooth solution shown in Figure 5 of $\mathrm{B} \& \mathrm{P}$.

The solution $\theta=t^{-1 / 4} z^{1 / 2} e^{-z / 2}$ (with $C_{1}=0, C_{2}=1$ in Eq. (3.5) above) gives $u=$ $(x / 2 t)-(2 / x)$ for (3.6) and corresponds to (3.5) of B \& $\mathrm{P}$.

(ii) Rodin [7] has considered the special case $n=0$ with

$$
Q(z)=-\left(a_{1} z^{1 / 2}+a_{2} z\right),
$$

where $a_{1}$ and $a_{2}$ are real constants. In Rodin's notation, $z$ stands for $x^{2} / t$. Eq. (3.4) can now be transformed into

$$
\frac{d^{2} \Psi}{d Y^{2}}+\frac{1}{2(1+2 \alpha)} Y \frac{d \Psi}{d Y}+\frac{\beta^{2}+2 \alpha}{4(2 \alpha+1)^{2}} \Psi=0
$$

where $\Psi=\exp \left(-\left(\alpha z+\beta z^{1 / 2}\right)\right) \psi, Y=2(2 \alpha+1) z^{1 / 2}+2 \beta$,

$$
\alpha=\frac{-1 \pm\left(1+4 a_{2}\right)^{1 / 2}}{2}, \quad \beta= \pm \frac{2 a_{1}}{\left(1+4 a_{2}\right)^{1 / 2}} .
$$

Obviously Eq. (3.7) admits erf solutions only if $\beta^{2}+2 \alpha=0$. Thus, there is an error in Rodin's solution (Eqns. (2.6) and (2.8) of his paper). However, under the condition 
$\beta^{2}+2 \alpha=0$, solutions similar to his in terms of error function are easily obtained. These correspond to (3.1) and (3.3) of $\mathrm{B} \& \mathrm{P}$.

(iii) On the other hand, for general $n$, Eq. (3.4) for the above $Q(z)$ can be put in the form

$$
\bar{Y} \frac{d^{2} \Psi}{d \bar{Y}^{2}}+\left(\frac{1}{2}-\bar{Y}\right) \frac{d \Psi}{d Y}-\frac{\beta^{2}+2 \alpha-4 n}{4(1+2 \alpha)} \Psi=0
$$

where

$$
\bar{Y}=\frac{-1}{4(1+2 \alpha)}\left[2(2 \alpha+1) z^{1 / 2}+2 \beta\right]^{2} .
$$

This is a confluent hypergeometric equation. It has solutions in terms of Hermite functions, so that

$$
\theta=t^{n} \exp \left(\alpha z+\beta z^{1 / 2}\right) H_{\nu}\left[2(2 \alpha+1) z^{1 / 2}+2 \beta\right],
$$

if $\alpha=-5 / 8, \beta=-8 a_{1}, \nu=2\left(64 a_{1}{ }^{2}-4 n-5 / 4\right)$ where $a_{1}$ may be chosen to make $\nu$ an integer. This solution gives

$$
u=\frac{x}{t}\left[\frac{5}{8}+4 a_{1} z^{-1 / 2}+\frac{1}{4} z^{-1 / 2} \frac{H_{\nu}{ }^{\prime}\left(-\frac{z^{1 / 2}}{2}-16 a_{1}\right)}{H_{\nu}\left(-\frac{z^{1 / 2}}{2}-16 a_{1}\right)}\right],
$$

corresponding to (3.4) of $\mathrm{B} \& \mathrm{P}$.

(iv) The Burgers-like equations (2.6) may directly admit similarity solutions. For example, for the equation

$$
u_{t}+u u_{x}=u_{x x}+R(t, x)
$$

(in the non-dimensional form) with $R=t^{-3 / 2} \bar{R}(\xi)$ and $\xi=z^{1 / 2}$, the similarity solution is given by the equation

$$
\frac{d^{2} G}{d \xi^{2}}-2 G \frac{d G}{d \xi}+2 \xi+2 \bar{R}(\xi)=0
$$

where $u=t^{-1 / 2}(G+\xi)$. This equation can be put in the standard Riccati form

$$
\frac{d G}{d \xi}-G^{2}=-2 \int[\xi+\bar{R}(\xi)] d \xi .
$$

These similarity solutions correspond to $\left(3.1^{\prime}\right)$ of $\mathrm{B} \& \mathrm{P}$, shown in their figures $9,10,11$, and describe solitary compression pulses, Lighthill [9].

b) Product solutions. Assuming $\theta=T(t) X(x)$, it is obvious that meaningful solutions exist only if $P$ is either a function of $x$ or of $t$ alone. The latter case leads merely to the homogeneous equations (2.6) with $R=0$. Therefore, only the case $P=P(x)$ needs to be considered. We obtain, with $T=e^{\lambda t}$ and $\lambda$ a constant, the following equation for $X$ :

$$
X^{\prime \prime}(x)+(P(x)-\lambda) X=0 .
$$

This equation is amenable to a very general discussion. However, we consider the special cases for $P=b x$ and $P=b x^{2}$ where $b$ is a constant. 
For the former, we have

$$
\theta=e^{\lambda t} Z^{1 / 2} J_{ \pm 1 / 3}\left(\frac{2}{3} Z^{3 / 2}\right), \quad Z=b^{-2 / 3}(b x-\lambda)
$$

which, for $b=-1$, becomes

$$
\theta=e^{\lambda t} \underset{B i}{A i}(\lambda+x)
$$

These solutions are similar to the solutions (1.1)-(1.6) of B \& P.

For $P=b x^{2}$, we can obtain a large number of solutions (Murphy [10]) expressible in terms of Weber-Hermite functions.

Finally, we note the family of solutions above, expressible in terms of Bessel or Hermite functions, may be made use of to solve a general initial-value problem; see Titchmarsh [11], Grinberg [12]. Such solutions will correspond to Table 4 of B \& P and can be made to provide solutions representing wave profiles having both positive and negative phases.

\section{REFERENCES}

[1] E. R. Benton and G. W. Platzman, A table of solutions of one-dimensional Burgers equation, Quart. Appl. Math. 30, 195-212 (1972)

[2] P. L. Sachdev and R. Seebass, Propagation of spherical and cylindrical N-waves, J. Fluid Mech. 58, 197-205 (1973)

[3] L. Sirovich and T. H. Chong, Supersonic flight in a stratified sheared atmosphere, Phys. Fluids 17, 310-320 (1974)

[4] N. N. Romanova, The vertical propagation of shorl acoustic waves in the real atmosphere, Izv. Atmospheric and Oceanic Physics 16, 134-145 (1970)

[5] J. D. Murray, Perturbation effects on the decay of discontinuous solutions of non-linear first order wave equations, SIAM J. Appl. Math. 19, 273-298 (1970)

[6] J. D. Murray, Singular perturbations of a class of non-linear hyperbolic and parabolic equations, J. Math. Phys. 47, 111-133 (1968)

[7] E. Y. Rodin, A Riccati solution for Burgers equations, Quart. Appl. Math. 27, 541-545 (1969)

[8] C. W. Chu, A class of reducible system of quasi-linear partial differential equations, Quart. Appl. Math. 23, 275-278 (1965)

[9] M. J. Lighthill, Viscosity effects in sound waves of finite amplitude, in Surveys in mechanics (G. K. Batchelor and R. M. Davies, editors), Cambridge Univ. Press, Cambridge, 1956, 250-351

[10] G. M. Murphy, Ordinary differential equations and their solutions (p. 327), D. Van Nostrand, Princeton, 1960

[11] E. C. Titchmarsh, Eigenfunction expansions, Part 1 (\$4.2, 4.12), Oxford Univ. Press, 1962

[12] G. A. Grinberg, On the temperature or concentration fields produced inside an infinile or finite domain by moving surfaces at which the temperature or concentration are given as functions of time, PMM 33, 1021 (1969) 